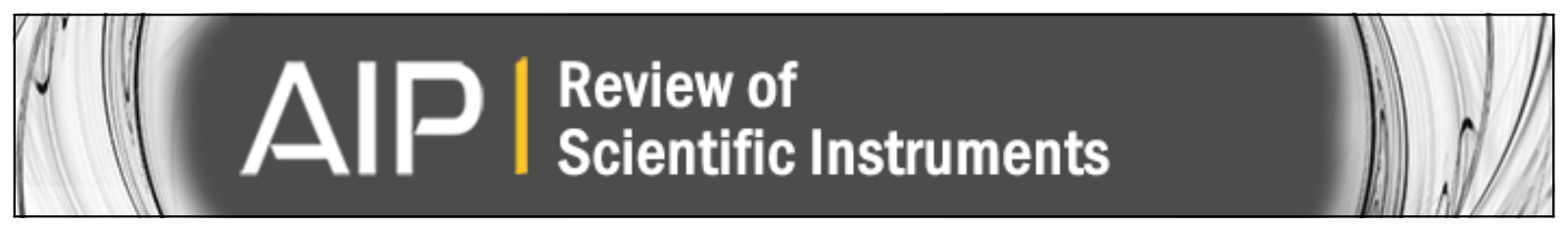

\title{
Device for filamentous fungi growth monitoring using the multimodal frequency response of cantilevers
}

N. Maloney, G. Lukacs, S. L. Ball, and M. Hegner

Citation: Review of Scientific Instruments 85, 015003 (2014); doi: 10.1063/1.4854655

View online: http://dx.doi.org/10.1063/1.4854655

View Table of Contents: http://scitation.aip.org/content/aip/journal/rsi/85/1?ver=pdfcov

Published by the AIP Publishing

\section{Articles you may be interested in}

Assessment of the inhibition of Dengue virus infection by carrageenan via real-time monitoring of cellular oxygen consumption rates within a microfluidic device

Biomicrofluidics 8, 024110 (2014); 10.1063/1.4870772

Piezoresistive measurement of Swine $\mathrm{H} 1 \mathrm{~N} 1$ Hemagglutinin peptide binding with microcantilever arrays

AIP Advances 4, 037118 (2014); 10.1063/1.4869636

High-throughput study of alpha-synuclein expression in yeast using microfluidics for control of local cellular microenvironment

Biomicrofluidics 6, 014109 (2012); 10.1063/1.3683161

Dielectrophoretic microfluidic device for the continuous sorting of Escherichia coli from blood cells

Biomicrofluidics 5, 032005 (2011); 10.1063/1.3608135

Origin of the response of nanomechanical resonators to bacteria adsorption

J. Appl. Phys. 100, 106105 (2006); 10.1063/1.2370507 


\title{
Device for filamentous fungi growth monitoring using the multimodal frequency response of cantilevers
}

\author{
N. Maloney, ${ }^{\text {a) }}$ G. Lukacs, S. L. Ball, and M. Hegnera) \\ Centre for Research on Adaptive Nanostructures and Nanodevices (CRANN), Trinity College Dublin, \\ Dublin 2, Ireland
}

(Received 19 November 2013; accepted 9 December 2013; published online 13 January 2014)

\begin{abstract}
Filamentous fungi cause opportunistic infections in hospital patients. A fast assay to detect viable spores is of great interest. We present a device that is capable of monitoring fungi growth in real time via the dynamic operation of cantilevers in an array. The ability to detect minute frequency shifts for higher order flexural resonance modes is demonstrated using hydrogel functionalised cantilevers. The use of higher order resonance modes sees the sensor dependent mass responsivity enhanced by a factor of 13 in comparison to measurements utilizing the fundamental resonance mode only. As a proof of principle measurement, Aspergillus niger growth is monitored using the first two flexural resonance modes. The detection of single spore growth within $10 \mathrm{~h}$ is reported for the first time. The ability to detect and monitor the growth of single spores, within a small time frame, is advantageous in both clinical and industrial settings. (C) 2014 AIP Publishing LLC. [http://dx.doi.org/10.1063/1.4854655]
\end{abstract}

\section{INTRODUCTION}

Microorganism growth detection and monitoring is of great importance in a range of microbiological fields. Microbial fermentation technologies require growth monitoring of both the fermenting microbe and any possible contaminants. ${ }^{1-3}$ In the pharmaceutical and food industries, culturing of bacteria and microfungi is an irreplaceable part of preservative efficiency testing procedures. ${ }^{4}$ Along with the industrial sector, detecting the presence of microbes rapidly and reliably is invaluable in clinical diagnostics. Detection and monitoring approaches are typically based on conventional microbiological methods (agar plate culturing and colony counting), which are time consuming and require highly skilled personnel. Additional techniques for microbe detection rely on the recognition of specific molecules in a sample. Polymerase Chain Reaction (PCR) based methods, while highly sensitive, ${ }^{5,6}$ require pre-existing knowledge of the characteristic nucleotide sequence of microbial agents, and also suffer from an inability to discriminate between living and dead cells in a label free manner. Detection of metabolic compounds can also provide useful information for growth monitoring of microbes in complex samples during fermentation processes. ${ }^{7}$ Newly developed growth detection and monitoring techniques are aimed towards fast, label free detection of viable cells with a portable hand held device being preferred.

Advancements in micro- and nanofabrication technologies have enabled the development of new techniques for microbe detection and growth monitoring. Examples of various biosensing techniques utilised for microbe detection are: evanescent field fibre optic sensor, ${ }^{8}$ surface Plasmon resonance (SPR), ${ }^{9}$ and mechanical sensors such as the quartz crystal microbalance (QCM) ${ }^{10}$ In these cases, carefully selected specific antibodies are used to detect microbes in a liquid environment. While highly specific, these techniques do not fa-

a)Electronic addresses: maloneni@tcd.ie and martin.hegner@tcd.ie cilitate live/dead cell discrimination or the monitoring of viable cell growth.

Micrometer sized cantilevers have been utilised as sensors in a range of fields since the early 1990s. ${ }^{11-14}$ Measurements are typically performed by tracking the static deflection (static mode), or resonance frequency (dynamic mode) of the sensors with respect to time. Measurement of cantilever motion can be performed using a variety of techniques. The most widely used techniques are laser beam deflection, ${ }^{15}$ piezoresistive, ${ }^{16}$ and piezoelectric ${ }^{17}$ readout. Several works have been published where cantilevers were used as biosensors in various biological fields. ${ }^{18-24}$ In the field of microbiology, studies have successfully demonstrated their use as mass sensors for pathogen detection via antibody-antigen chemistry. ${ }^{25-27}$ Techniques moving away from antibody-antigen immobilisation have also been reported with the detection of yeast cells (Saccharomyces cerevisiae (S. cerevisiae)) using poly-L-lysine, ${ }^{28}$ and the detection of Escherichia coli (E. coli) using cantilevers functionalised with mannosides. ${ }^{29}$ These techniques also suffer from live/dead cell discrimination issues.

When cantilevers are utilised for real time detection and monitoring of viable microorganisms, additional considerations must be made. These are: appropriate surface functionalisation, and the incorporation of carefully chosen in situ reference sensors in measurements to ensure that false signals are eliminated. The sensor surface should provide optimal growth conditions without detachment of the microorganism as reported in Refs. 30-32 where antibodies are used to attach microbes to the cantilevers surface. Detachment can cause misinterpretation of growth or cross contamination when measurements are performed in a liquid environment. ${ }^{33}$ Performance of growth measurements in humid air removes the need for antibody attachment or trapping of microbes on the sensor, hence simplifying cantilever functionalisation. ${ }^{34,35}$ Microcantilevers in these measurements serve as miniature Petri dishes where nutrition is retained in a hydrogel on the cantilever's surface. By changing the applied nutrition this 
method can be adapted to the culture based growth detection of any microbe. The utilisation of arrays containing eight cantilevers allows for multiple growth measurements to be performed in parallel, while also allowing for the provision of in situ reference sensors.

Here, we present a newly developed device for the monitoring of microorganism growth using cantilever arrays. The device is capable of measuring multiple resonance modes of several cantilevers in a time multiplexed fashion. As a proof of principle, the growth of single A. niger spores is monitored using the fundamental and second flexural resonance modes of cantilevers in an array.

\section{EXPERIMENTAL}

\section{A. Instrument development}

Measurement of cantilever resonance frequencies is via the optical beam deflection method. All optical components were purchased from Thorlabs (Cambridgeshire, UK) unless otherwise stated. A single wavelength $(\lambda=830 \mathrm{~nm})$ pigtailed laser diode (LPS-830-FC) accompanied by a collimator is used to provide a collimated laser beam (beam diameter $=3.45 \mathrm{~mm}$ ) to the system. The laser diode is driven with an external module (ITC502-Benchtop Laser Diode and Temperature Controller) and is temperature controlled (TCLDM9TE-Cooled Mount) to ensure output stability. A convex lens (focal length $=30 \mathrm{~mm}$ ) is utilised to focus a laser spot on the cantilever. The resultant laser spot has a diameter and depth of focus of $\sim 9 \mu \mathrm{m}$ and $\sim 425 \mu \mathrm{m}$, respectively. The input optical axis is maintained using a $16 \mathrm{~mm}$ cage system comprised of several components: cage plates (SP03), cage rods (SR3), quick release cage mounts (QRC05A), collimator adaptor (AD1109F), cage mounting bracket (SMB1), and several retaining rings (SM05RR).

Two automated translation stages (M-110K043, Physik Instrumente (PI), Bedford, UK) arranged orthogonally (one horizontal and one vertical) facilitate movement of the laser spot across and along the cantilever array. In order for the stage to operate at high relative humidity $(\mathrm{RH})$ levels $(\sim 95 \%)$, the encoder has been insulated from the environment. These stages have a travel range of $5 \mathrm{~mm}$, a minimum incremental motion of $50 \mathrm{~nm}$, and a unidirectional repeatability of $0.1 \mu \mathrm{m}$ which ensures that the laser spot is returned to the desired position, and that no additional noise is added to dynamic mode measurements. Automated control of the translation stages is performed via a PI PCI computer board (C-843 Motor Controller Card). The vertical translation stage acts as the mount for the optical cage system which provides the input laser beam for the device. A xyz micro-translation stage (Gothic arch 9061-XYZ, Newport, CA, USA) is mounted on the horizontal translation stage and acts as the mount for the vertical translation stage. The micro-translation stage is used for initial positioning of the laser spot onto cantilevers.

Cantilever arrays are mounted on a Polyether ether ketone (PEEK) holder containing a piezoelectric ceramic stack. This stack is used to actuate cantilevers at their resonance frequencies. Torrseal ${ }^{\circledR}$ is employed to ensure electrical shorting of the piezoelectric ceramic stack does not occur during oper- ation at high RH levels. The cantilever array is held in position using a PEEK clamp.

A $10 \times 10 \mathrm{~mm}$ 1D PSD (SI-0299, Laser Components Ltd., UK) with accompanying signal processing circuitry (SPC) (SPC02, Laser Components Ltd., UK) on its backside is used for measurement of flexural resonance modes. The bandwidth of the PSD-SPC electronics is $400 \mathrm{kHz}$. The PSD is housed in a customised holder to protect the SPC from high humidity. The pins on the PSD are inserted into 2 PCI brackets to allow readout of electrical signals. For insulation from the external humid environment, an O-ring and two pieces of gasket sealer are employed. An illustration of the entire device assembly is shown in Figure 1(b).

Frequency measurements for each cantilever in an array are performed sequentially. As the beam deflection method is sensitive to angular deflections, the laser spot is moved to a node of vibration using the 2D translation stage system. This ensures that amplitude spectra with good signal to noise ratios are recorded. An arbitrary waveform generator (NI-PCI-5412, National Instruments, TX, USA) provides a sinusoidal linear frequency sweep for actuation of the cantilevers in the array. The resulting signal, generated by motion of the deflected laser beam on the PSD, is converted to a differential voltage by the SPC. This signal is preamplified (SR560 Low Noise Preamplifier, Stanford Research Systems, CA, USA) and digitised (NI-PCI-5112, National Instruments, TX, USA), before being compared to the signal from the frequency generator to produce amplitude and phase spectra. In order to satisfy Nyquist's theorem, a sampling rate of $10^{6}$ samples/s is used. After a frequency scan has been performed, the dual translation stage moves to a node of vibration on an adjacent cantilever (the position of which has been predetermined), and the above is repeated for each cantilever in an array. This can be repeated for multiple modes of vibration. A schematic of device operation is shown in Figure 1(a).

The device is housed in a custom designed environmental chamber (5500-8139 A, Electro Tech Systems, Inc., PA, USA), which is used to regulate temperature and humidity. Stable temperatures are generated using a heating element housed inside the chamber. Humid air is pumped into the chamber using an ultrasonic humidification system (5472-3, Electro Tech Systems, Inc., PA, USA). Humidity and temperature are regulated using a temperature compensated humidity sensor (554, Electro Tech Systems, Inc., PA, USA) in conjunction with a microprocessor controller (5200 441-431, Electro Tech Systems, Inc., PA, USA). This system allows humidity and temperature levels to be kept within $\pm 0.5 \%$ and $\pm 0.2^{\circ} \mathrm{C}$ of the desired set points, respectively. This stability is crucial for the proper analysis of the sensors nanomechanical response.

\section{B. Materials and methods}

All chemicals and reagents are of analytical grade and were purchased from Sigma-Aldrich (Arklow, Ireland), unless otherwise stated. In the measurements to be presented, Si (001) cantilever arrays (IBM Research Laboratory, Rüschlikon, Switzerland) were used. Arrays consist of eight 


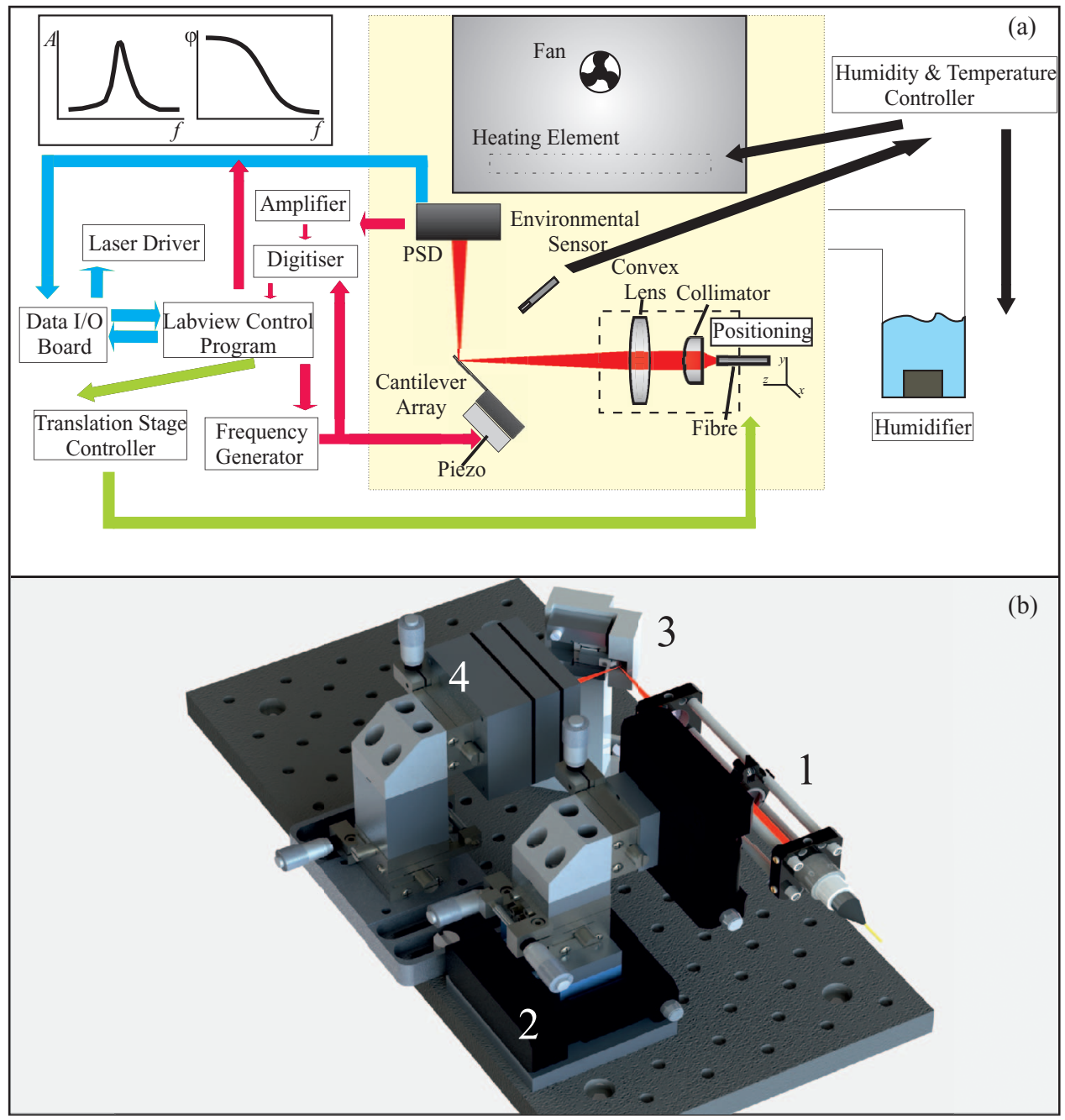

FIG. 1. (a) Schematic of cantilever array device. LabVIEW is used to control the dynamic mode operation (red arrows), output laser power (blue arrows), and translation stage motion (green arrows). Environmental control is provided via an external microprocessor controller (black arrows). For the dynamic mode operation, an IR laser beam $(\lambda=830 \mathrm{~nm})$ is focused onto a cantilever. To record a resonance spectrum, the laser spot is moved to a position along a cantilever which provides the best signal to noise ratio for the desired flexural resonance mode. The frequency generator board provides a sinusoidal frequency signal to the piezoelectric actuator. The movement of the laser spot on the PSD is converted to a voltage and is preamplified before being recorded by the digitiser board to produce frequency and phase spectra. The laser spot is then moved to an adjacent cantilever in the array using the dual translation stage setup and the process is repeated. (b) Rendered image of device. (1) Input optics cage system mounted on vertical translation stage. A collimated laser beam is focused to a spot via a convex lens. The vertical translation stage facilitates movement of the laser spot to cantilevers in an array. (2) Horizontal automated translation. This stage allows motion along the cantilever bar so spectra with good signal to noise ratios are obtained. (3) Piezoelectric cantilever mount. The cantilever array is mounted on a PEEK holder containing a piezoelectric ceramic stack. This stack is used to actuate cantilevers at their resonance frequencies. (4) PSD in environmental protective housing. A $10 \times 10 \mathrm{~mm}$ 1D PSD with accompanying SPC on its backside is used for measurement of flexural resonance modes. The bandwidth of the PSD-SPC electronics is $400 \mathrm{kHz}$. The PSD is housed in a custom holder to protect the SPC from high RH.

cantilevers separated by a pitch of $250 \mu \mathrm{m}$. Each cantilever has a length and width of $500 \mu \mathrm{m}$ and $100 \mu \mathrm{m}$, respectively. The cantilevers used in these measurements have a thickness of either $7 \mu \mathrm{m}$ or $2 \mu \mathrm{m}$.

A. niger (CIP 1431.83) spores were obtained from the Pasteur Institute (Paris, France). Spores were cultured on potato dextrose agar (PDA) (Oxoid, Basel, Switzerland) slants at $35^{\circ} \mathrm{C}$ for five days and then stored at $4{ }^{\circ} \mathrm{C}$ until required.

\section{Multimode response of agarose hydrogel functionalised cantilevers}

Cantilever arrays were cleaned by exposure to oxygen plasma for 3 min using the following settings: 0.3 mbar $_{2}$,
160 W, 40 kHz (PICO Barrel Asher, Diener electronic GmbH + Co. KG, Ebhausen, Germany). The arrays were immediately immersed in a silane solution (3-glycidyloxypropyltrimethoxysilane:N-ethyldiisopropylamine:water free toluene: 1:1:100) for $45 \mathrm{~min}$ and then washed twice in water free toluene for $15 \mathrm{~min}$. The arrays were dried using nitrogen gas. This step provides an epoxy activated surface which is reactive in alkali conditions towards primary hydroxyl groups found in agarose. The $\mathrm{pH}$ of an agarose (SeaKem Gold Agarose, Bioconcept, New Hampshire, USA) solution ( $1 \% \mathrm{wt} . / \mathrm{vol}$.) was adjusted to $\sim 11.9$ by addition of $2 \mathrm{M} \mathrm{NaOH}$ to facilitate the epoxy coupling. The cantilevers were functionalised by immersion in heated capillary tubes $\left(\sim 100^{\circ} \mathrm{C}\right)$, containing the $\mathrm{pH}$ adjusted agarose solution, for $3 \mathrm{~s}$. 
The $2 \mu \mathrm{m}$ thick agarose functionalised cantilever array was mounted on the piezo actuator and the laser system was aligned. The temperature in the chamber was set to $30^{\circ} \mathrm{C}$ and allowed to stabilise for $30 \mathrm{~min}$. The relative humidity was ramped slowly from $\sim 75 \%$ to $\sim 90 \%$ and then held at this final value for several hours. Resonance frequency data were recorded for the first four resonance modes. For each mode, 2000 data points were recorded with a sampling time of $2 \mathrm{~ms}$ per data point. A full scan of the array was repeated every 3 min.

An additional test was performed using the $7 \mu \mathrm{m}$ thick agarose functionalised cantilever array. The array was placed in the chamber and the temperature was set to $30^{\circ} \mathrm{C}$. RH levels were ramped from $86 \%$ to $96 \%$ and allowed to return to $86 \%$ at a period of $\sim 25 \mathrm{~min}$. The response of the first flexural resonance mode was recorded. 400 data points were taken per spectrum with a sampling time of $6 \mathrm{~ms}$ per data point. A full scan of the array was taken every 2 min.

\section{Multimode Aspergillus niger growth detection and monitoring}

A. niger (CIP 1431.83) spores were harvested and suspended in $7 \mathrm{ml}$ of $0.01 \%$ tween solution. $100 \mu 1$ of this suspension was pipetted onto a sterile glass cover slip and left to dry.

A $7 \mu \mathrm{m}$ thick cantilever array was agarose functionalised as described above. The array was immersed in Roswell Park Memorial Institute medium broth (RPMI-1640), which was supplemented with $0.165 \mathrm{M}$ MOPS $(\mathrm{pH}=7)$ and $0.2 \%$ glucose, for $10 \mathrm{~min}$ in order to load the agarose layer with nutrition. A glass needle on a micro-manipulator was used to transfer individual spores from the glass cover slip onto the front end of a cantilever. This was performed for two cantilevers in the array.

Environmental conditions inside the device chamber were set to $94 \% \mathrm{RH}$ and $30^{\circ} \mathrm{C}$ and allowed to equilibrate for $>30 \mathrm{~min}$. After spore deposition the cantilever array was mounted on the piezoelectric actuator and placed into the device. The fundamental and second flexural resonance modes were recorded for cantilevers in the array. 400 data points with a sampling time of $8 \mathrm{~ms}$ per point and 1000 data points with a sampling time of $2 \mathrm{~ms}$ were recorded for the fundamental and second flexural resonance modes, respectively. A full scan of the cantilever array was repeated every $30 \mathrm{~min}$.

\section{RESULTS AND DISCUSSION}

\section{A. Multimode resonance spectra}

The resonance spectrum, with accompanying phase $(\varphi)$ shift (blue), of 2 (black) and 7 (red) $\mu \mathrm{m}$ thick cantilevers is shown in Figure 2. In the case of the $2 \mu \mathrm{m}$ thick cantilever, the first four flexural resonance modes are shown, while the first two modes are shown for the $7 \mu \mathrm{m}$ thick cantilever. The phase spectrum for the $7 \mu \mathrm{m}$ thick cantilever has been omitted for clarity. The spectra consist of 2000 data points and took $4 \mathrm{~s}$ each to obtain. In order to visualise the fundamental resonance mode for the $2 \mu \mathrm{m}$ thick cantilever (shown in the

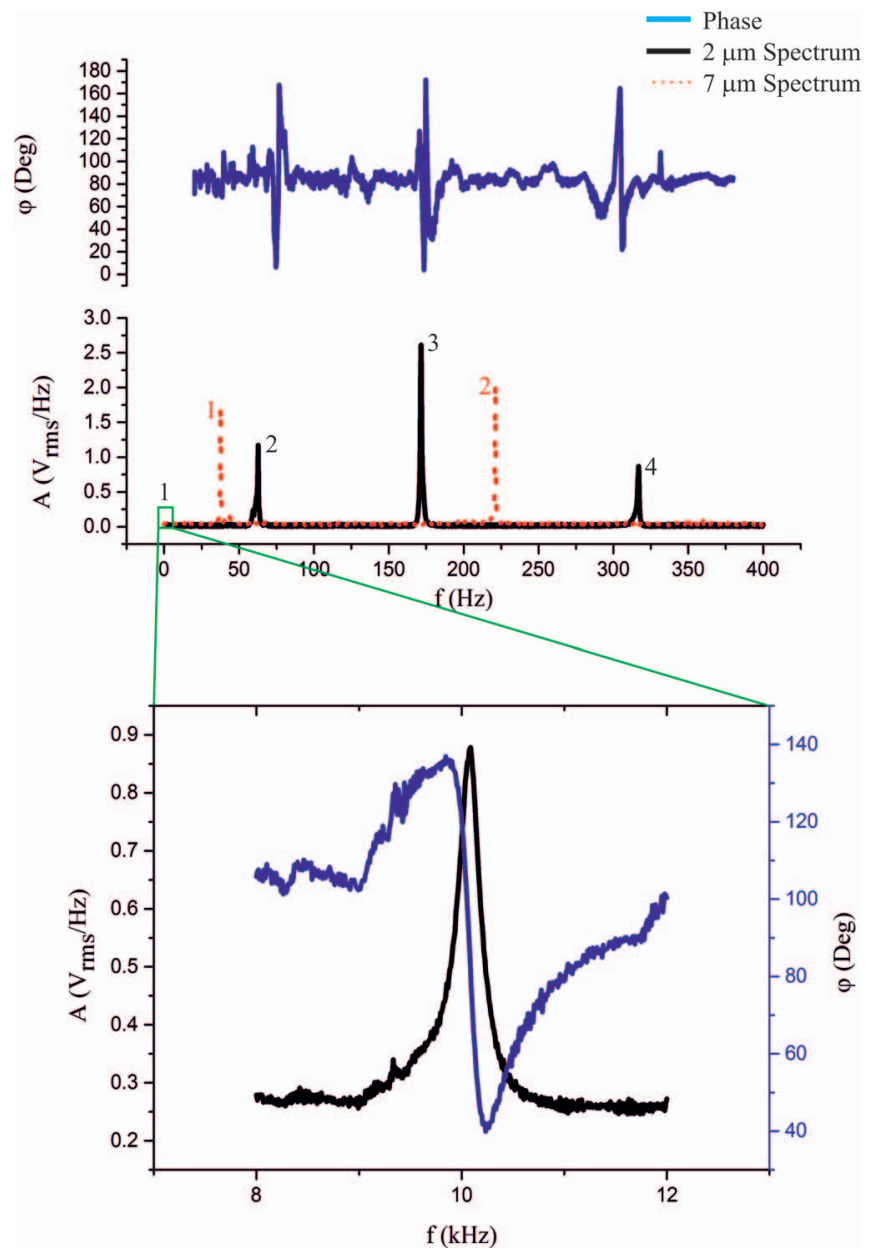

FIG. 2. The resonance spectrum, with accompanying phase $(\varphi)$ shift (blue), of 2 (black) and 7 (red) $\mu \mathrm{m}$ thick cantilevers. The first four flexural resonance modes are shown in the case of the $2 \mu \mathrm{m}$ cantilever. The first two flexural resonance modes are shown in the case of the $7 \mu \mathrm{m}$ cantilever. The phase spectrum for the $7 \mu \mathrm{m}$ has been omitted for clarity.

expanded view in Figure 2), a higher voltage must be applied to the piezoelectric actuator. Movement of the laser spot to the individual resonance nodes allows multiple resonance modes to be recorded for each cantilever in an array. Using a previous iteration of this device, ${ }^{34}$ measurement of higher modes of vibration, with fibre optical means, was not possible which limited the achievable sensitivity of measurements.

\section{B. Multimode response of agarose functionalised cantilevers}

The change in flexural resonance frequency of the first four modes of a $2 \mu \mathrm{m}$ cantilever is shown in Figure 3(a2). Changes in resonance frequency are due to the absorption of water from the surrounding humid environment by the agarose layer on the cantilever. The black, red, green, and blue plots correspond to the fundamental, second, third, and fourth resonance modes, respectively. There is a direct correlation between humidity levels (Figure 3(a1); orange plot) and the resonance frequency of an agarose coated cantilever. For the cantilever shown, a mass responsivity (mass sensitivity ${ }^{-1}$ ) of $38.8 \pm 0.8,10.3 \pm 0.1,4.3 \pm 0.1$, and $2.9 \pm 0.1 \mathrm{pg} / \mathrm{Hz}$ is 

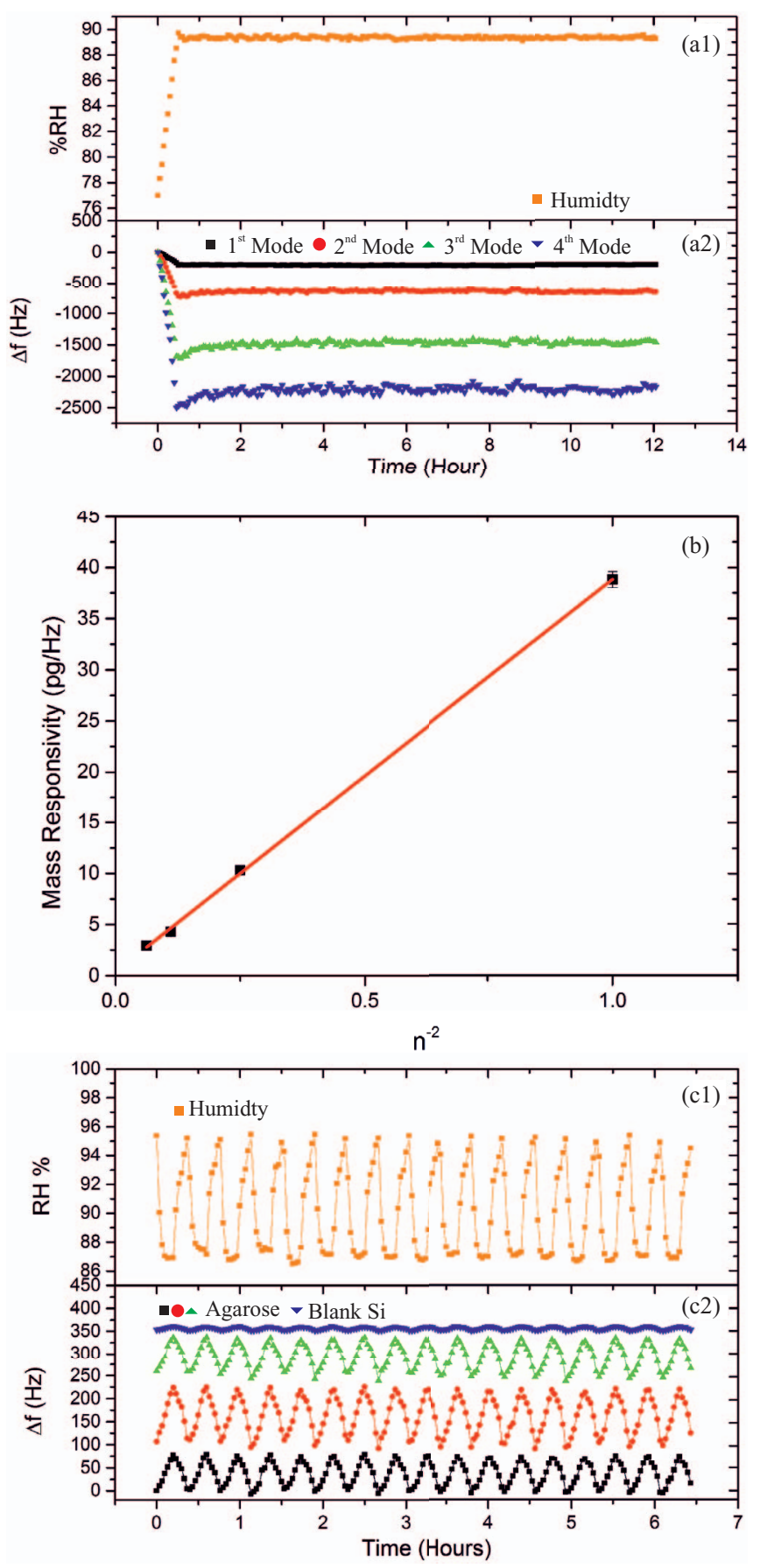

FIG. 3. (a1) Relative humidity. (a2) Flexural resonance frequency response of the first four modes of a $2 \mu \mathrm{m}$ thick agarose functionalised cantilever due to varying RH conditions. Use of higher resonance modes increases device sensitivity by a factor of 13 when compared to the fundamental resonance mode. (b) Plot of $\mathrm{n}^{-2}$ versus mass responsivity. There is a linear increase in sensitivity with mode number squared. (c1) Controlled cycling of relative humidity. (c2) Fundamental mode response of one blank Si cantilever (blue) and 3 agarose coated cantilevers (green, red, and black) on a $7 \mu \mathrm{m}$ thick array.

revealed for the fundamental, second, third, and fourth flexural resonance modes, respectively, assuming a uniform coverage of agarose on the cantilever's surface. The ability to record the fourth resonance mode has increased the achievable sensor dependent mass sensitivity of the device by a factor of 13 when compared to the fundamental resonance mode. Figure 3(b) shows inverse mode number squared $\left(\mathrm{n}^{-2}\right)$ ver- sus mass responsivity. There is a linear relationship between square of mode number and sensitivity which is in agreement with data published. ${ }^{36}$ This demonstrates the ability of this device to accurately record the multimode resonance frequency response of cantilevers in an array. The data shown in Figure 3(a1), between the time points of 2 and $12 \mathrm{~h}$, demonstrates the excellent environmental control provided by the environmental chamber. This is essential for accurate growth detection measurements. It should also be noted that there is no resonance frequency drift over time for all modes.

Figure 3 shows an induced periodic fluctuation of humidity (c1) and the corresponding first mode frequency response of one blank $\mathrm{Si}$ cantilever and 3 agarose coated cantilevers on a $7 \mu \mathrm{m}$ thick array (c2). A direct correlation can be seen between humidity levels in the environmental chamber and the resonance frequency response of cantilevers. This figure demonstrates the following: (i) Water absorbed by an agarose layer is immediately lost as humidity levels surrounding the cantilevers decrease resulting in a rise in resonance frequency. (ii) The device is capable of measuring the resonance frequencies of cantilevers in varying conditions.

The results described in this section indicate that this new device is capable of recording resonance spectra for multiple flexural resonance modes in dynamically changing conditions. Operation of this device has also been demonstrated at the high $\mathrm{RH}$ levels required for growth measurements (>94\%).

\section{Multimode Aspergillus niger growth detection and monitoring}

The hypha growing fungus $A$. niger was used as a model organism to demonstrate the ability of this device to monitor microbial growth. After germination fungal conidia grow hyphal filaments which play a crucial role in colonisation of a substrate. The mechanical response of agarose coated cantilevers was used as an in situ reference. The fundamental and second mode frequency response, due to A. niger growth, of two agarose functionalised cantilevers (sensor 1 and sensor 2 ) is shown in Figure 4. Illustrations of the vibrational mode shape for the fundamental and second resonance modes, along with red dots to indicate the position of the laser spot during frequency measurements, are also shown. Optical microscope images of the top and back sides of the cantilevers in question are shown in Figure 5. To ease visualisation, hyphal growth on each cantilever has been highlighted. Hyphal growth is highlighted using different colours which correspond to the colours of the plots in Figure 4.

It can be seen (Figure 4) that for both modes there is a discernible change in resonance frequency within $10 \mathrm{~h}$, indicating the detection of viable spore growth. This time is an order of magnitude faster than conventional methods currently used in clinical or industrial settings. The detection time here is comparable to those which have been previously published using cantilevers. ${ }^{34}$ It should be noted that the rapid detection of the growth of single A. niger spores has been reported for the first time. The ability to detect the growth of a small inoculum within a short time is advantageous, especially in a clinical setting. Hyphal growth is monitored for more than $24 \mathrm{~h}$ as 

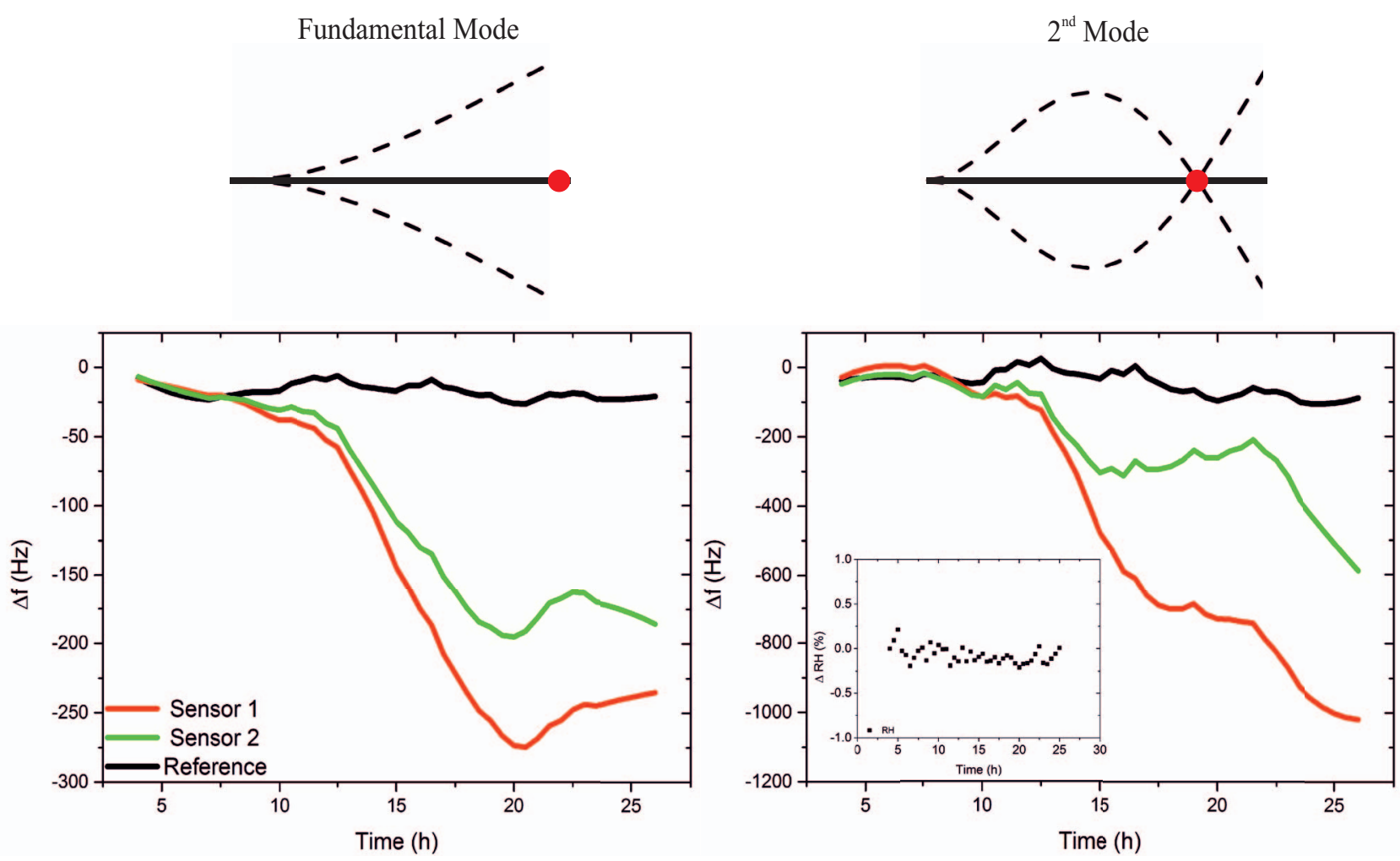

FIG. 4. Fundamental (left side) and second mode (right side) frequency response, due to A. niger spore growth, of 2 spore and agarose functionalised cantilevers (red (sensor 1) and green (sensor 2) curves). Agarose functionalised cantilevers were used as reference sensors. Black line is an average of several reference sensors. The plots shown have been smoothed using a Savitzky-Golay filter. Changes in resonance frequency indicate the detection of viable A. niger growth within $10 \mathrm{~h}$. The inlayed plot shows stability of RH conditions during the measurement. Vibrational mode shape for fundamental and 2nd modes are illustrated at top of the figure. The position of the laser spot for frequency measurement for each mode is also indicated (red dots).

indicated by the positive and negative frequency shifts which are observed (Figure 4). These frequency shifts are indicative of the propagation of hyphal growth along the cantilevers longitudinal axes. Cantilevers are traditionally thought of as mass sensors where a negative frequency shift is observed upon adsorption of mass on the cantilever. The group of Tamayo have published several works outlining the effects of adsorption position on the resonance response of cantilevers. ${ }^{37-39}$ In these works, it has been shown that the deposition of bacterial cells can produce both negative ("mass effect") and positive ("stiffness effect") frequency shifts. It is proposed that the stiffness (Young's modulus) of the adsorbed particle alters the flexural rigidity of the cantilever to produce positive frequency shifts. It has been shown that the "mass" and "stiffness" effects are proportional to the square of the amplitude of vibration and the curvature of the vibrational mode shape at the position, along the cantilever's longitudinal axis, of the adsorbed particle, respectively. Hence, as hyphal growth propagates along a cantilever's length different magnitudes and signs of frequency response are to be expected. For example, in the case of the fundamental resonance mode the positive frequency shifts observed around $20 \mathrm{~h}$ indicate that hyphal growth is occurring in the vicinity of the vibrational node where the stiffness effect dominates. In the case of sensor 2 , the subsequent decrease in resonance frequency is due to growth occurring in a mass sensitive region on the topside of the cantilever. Similar observations can be made for the 2 nd resonance mode. The magnitude of a positive frequency shift is indicative of the stiffness of the biological material on the cantilever. Thus, through monitoring of cantilever resonance frequencies

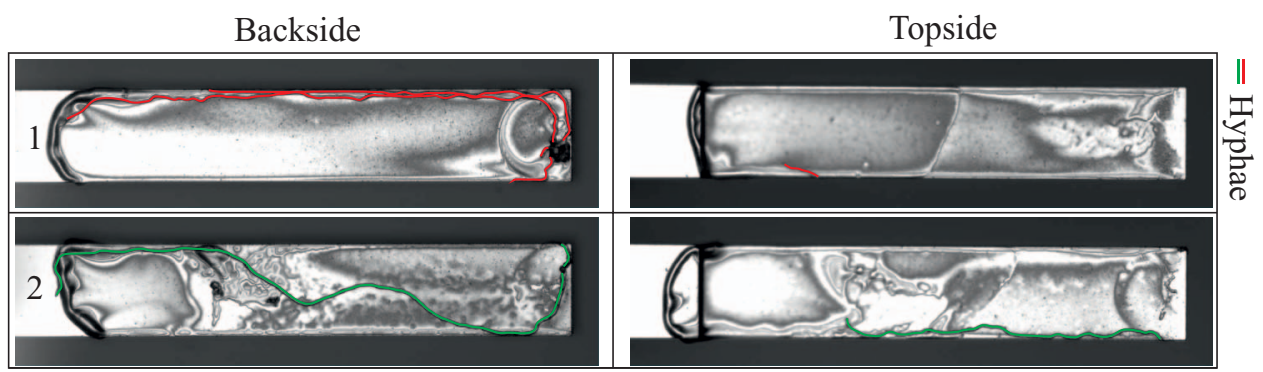

FIG. 5. Microscope images of A. niger growth on the top and bottom of sensors 1 and 2. These images correspond to the plots shown in Figure 4. Images were recorded at the end of the experiment. Hyphal growth has been coloured to aid visualisation. 


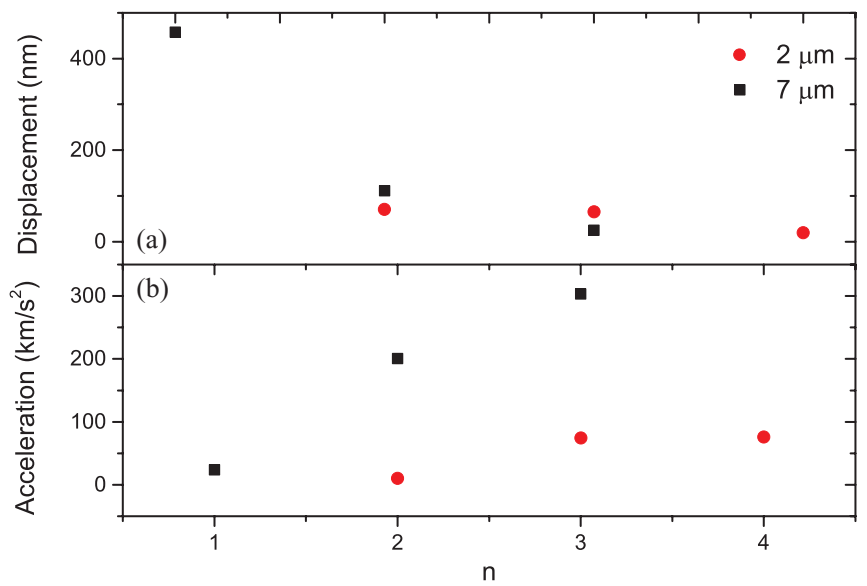

FIG. 6. (a) Maximum flexural displacement of a cantilever when oscillating at resonance for several modes of vibration. (b) Maximum flexural acceleration of cantilever when oscillating at resonance for several resonance modes. Data are shown for 2 and $7 \mu \mathrm{m}$ thick cantilevers. Data points represent average values of several measurements. Resonance frequencies for the $7 \mu \mathrm{m}$ thick cantilever are: $1 \mathrm{st}=36.1 \mathrm{kHz}, 2 \mathrm{nd}=214.2 \mathrm{kHz}$, and $3 \mathrm{rd}=552.1 \mathrm{kHz}$. Resonance frequencies for the $2 \mu \mathrm{m}$ thick cantilever are: $2 \mathrm{nd}=61.9 \mathrm{kHz}$, $3 \mathrm{rd}=169.3 \mathrm{kHz}$, and $4 \mathrm{th}=312 \mathrm{kHz}$. Measurements were performed using a laser Doppler vibrometer.

structural and mechanical changes in the biological material on the cantilever can be determined.

While providing no decrease in growth detection times the use of higher vibrational modes does provide extra information in regards to the mechanical properties of a growing hypha. When measuring with higher vibrational modes, the forces due to acceleration to which spores are exposed to increase (Figure 6(b)). Hence, the use of higher modes of vibration (e.g., $\mathrm{n}=3,4$ ) may have an effect on spore viability. Acceleration and displacement data were obtained using a laser Doppler vibrometer (Polytec Fibre Vibrometer OFV552, Polytec Ltd., LambdaHouse, BatfordMill, UK) for cantilevers with identical dimensions to those used in the experiments previously described. It is worth noting that the displacement to which the spores are exposed (in comparison to their size) is greatly reduced when operating at higher modes of vibration (Figure 6(a)). This reduces the effect that forces due to acceleration may have. The use of thinner cantilevers also reduces the acceleration to which the spores are subjected to during cantilever oscillation. For example, in the case of the third vibrational mode, acceleration for a $2 \mu \mathrm{m}$ thick cantilever is four times smaller than that of a $7 \mu \mathrm{m}$ thick cantilever while displacement is roughly the same (Figure 6). This can be explained by the fact that thinner cantilevers have lower resonance frequencies for a given vibrational mode. Hence, for multiple mode measurements use of thinner cantilevers will reduce any negative effects which cantilever oscillations may have on spore viability.

\section{CONCLUSION}

Due to its multicellular hyphal structure A. niger is an ideal model organism for cantilever measurements as detected growth events are easily validated through optical inspection once experiments are concluded. A previously described de- vice for microbe growth measurements, using cantilevers operated in dynamic mode, employed eight optical fibres aligned with eight measurement sensors. ${ }^{34}$ This setup did not allow for the measurement of higher vibrational modes but an automated measurement was possible when compared to earlier versions of the device. ${ }^{30-32,35}$ Measurement of higher vibrational modes is desirable as it greatly increases the sensitivity of measurements. ${ }^{36}$ The device presented here is capable of the accurate and reliable measurement of multiple resonance modes of cantilevers in an array. The readout of any array up to a size of $5 \times 5 \mathrm{~mm}$ (range of translation stages) is possible. The measurement of the first four flexural resonance modes is presented. For an agarose functionalised sensor $(500 \times 100$ $\times 2 \mu \mathrm{m}$ ), a mass responsivity of $2.9 \pm 0.1 \mathrm{pg} / \mathrm{Hz}$ is revealed, assuming a uniform absorption of water by the agarose layer along the cantilever's length. The ability to record the fourth resonance mode has enhanced the achievable sensitivity by a factor of 13 when compared to the fundamental mode. It should be noted that the dimensions of the particular cantilever sensor used determines the achievable sensitivity. The development of a device capable of measuring higher resonance modes allows for lower sensitivities to be realised.

The monitoring of single $A$. niger spore growth using the multimodal frequency response of two cantilevers is also presented. The detection of growth of viable spores has been shown within $10 \mathrm{~h}$ in the case of all sensors. This is an order of magnitude faster than conventional growth detection methods. The detection of the growth of single A. niger spores has been shown for the first time, demonstrating the high sensitivity and suitability of this device for microorganism growth detection. Using different environmental settings and growth media, according to the needs of other microorganisms, provides the opportunity to use this experimental platform as a general tool for fast microorganism growth monitoring. In order to gain a further understanding of the frequency response due to growth of hyphae on the cantilever surface, online imaging of the measurement will be required.

\section{ACKNOWLEDGMENTS}

This work was supported by Science Foundation Ireland under the CSET scheme (SFI08/CE/I1432) and PI scheme (09/IN.1/B2623).

\footnotetext{
${ }^{1}$ T. Sue, V. Obolonkin, H. Griffiths, and S. G. Villas-Bôas, Appl. Environ. Microbiol. 77(21), 7605-7610 (2011).

${ }^{2}$ L. De Vuyst, G. Vrancken, F. Ravyts, T. Rimaux, and S. Weckx, Food Microbiol. 26(7), 666-675 (2009).

${ }^{3}$ R. Van der Meulen, I. Scheirlinck, A. Van Schoor, G. Huys, M. Vancanneyt, P. Vandamme, and L. De Vuyst, Appl. Environ. Microbiol. 73(15), 47414750 (2007).

${ }^{4}$ C. L. Moser and B. K. Meyer, Am. Assoc. Pharm. Sci. 12(1), 222-226 (2011).

${ }^{5}$ T. K. Kourkoumpetis, B. B. Fuchs, J. J. Coleman, A. Desalermos, and E. Mylonakis, Clin. Infect. Dis. 54(9), 1322-1331 (2012).

${ }^{6}$ C. D. Sibley, G. Peirano, and D. L. Church, Infect. Genet. Evol. 12(3), 505-521 (2012).

${ }^{7}$ D. B. Kell, M. Brown, H. M. Davey, W. B. Dunn, I. Spasic, and S. G. Oliver, Nat. Rev. Microbiol. 3(7), 557-565 (2005).

${ }^{8}$ K. Rijal, A. Leung, P. M. Shankar, and R. Mutharasan, Biosens. Bioelectron. 21(6), 871-880 (2005)
} 
${ }^{9}$ A. D. Taylor, J. Ladd, Q. Yu, S. Chen, J. Homola, and S. Jiang, Biosens. Bioelectron. 22(5), 752-758 (2006).

${ }^{10}$ M. A. Cooper and V. T. Singleton, J. Mol. Recognit. 20(3), 154-184 (2007).

${ }^{11}$ J. L. Arlett, E. B. Myers, and M. L. Roukes, Nat. Nanotechnol. 6(4), 203 215 (2011)

${ }^{12}$ A. Boisen, S. Dohn, S. S. Keller, S. Schmid, and M. Tenje, Rep. Prog. Phys. 74(3), 036101 (2011).

${ }^{13}$ M. Calleja, P. M. Kosaka, A. San Paulo, and J. Tamayo, Nanoscale 4(16), 4925-4938 (2012).

${ }^{14}$ J. Tamayo, P. M. Kosaka, J. J. Ruz, A. San Paulo, and M. Calleja, Chem. Soc. Rev. 42(3), 1287-1311 (2013).

${ }^{15}$ G. Meyer and N. M. Amer, Appl. Phys. Lett. 53(12), 1045-1047 (1988)

${ }^{16}$ A. Boisen, J. Thaysen, H. Jensenius, and O. Hansen, Ultramicroscopy 82(1-4), 11-16 (2000).

${ }^{17}$ T. Itoh and T. Suga, Nanotechnology 4(4), 218 (1993).

${ }^{18}$ Y. Arntz, J. D. Seelig, H. P. Lang, J. Zhang, P. Hunziker, J. P. Ramseyer, E. Meyer, M. Hegner, and C. Gerber, Nanotechnology 14(1), 86-90 (2003).

${ }^{19}$ N. Backmann, C. Zahnd, F. Huber, A. Bietsch, A. Pluckthun, H. P. Lang, H. J. Guntherodt, M. Hegner, and C. Gerber, Proc. Natl. Acad. Sci. U.S.A. 102(41), 14587-14592 (2005).

${ }^{20}$ J. Fritz, M. Baller, H. Lang, H. Rothuizen, P. Vettiger, E. Meyer, H.-J. Güntherodt, C. Gerber, and J. Gimzewski, Science 288(5464), 316-318 (2000).

${ }^{21}$ F. Huber, H. Lang, N. Backmann, D. Rimoldi, and C. Gerber, Nat. Nanotechnol. 8(2), 125-129 (2013).

${ }^{22}$ J. Mertens, C. Rogero, M. Calleja, D. Ramos, J. A. Martín-Gago, C. Briones, and J. Tamayo, Nat. Nanotechnol. 3(5), 301-307 (2008).

${ }^{23}$ J. Zhang, H. Lang, F. Huber, A. Bietsch, W. Grange, U. Certa, R. McKendry, H.-J. Güntherodt, M. Hegner, and C. Gerber, Nat. Nanotechnol. 1(3), 214-220 (2006).

${ }^{24}$ G. Longo, L. Alonso-Sarduy, L. M. Rio, A. Bizzini, A. Trampuz, J. Notz, G. Dietler, and S. Kasas, Nat. Nanotechnol. 8(7), 522-526 (2013)
${ }^{25}$ A. Gupta, D. Akin, and R. Bashir, J. Vac. Sci. Technol. B 22(6), 2785-2791 (2004).

${ }^{26}$ B. Ilic, D. Czaplewski, H. G. Craighead, P. Neuzil, C. Campagnolo, and C. Batt, Appl. Phys. Lett. 77(3), 450-452 (2000).

${ }^{27}$ B. Ilic, D. Czaplewski, M. Zalalutdinov, H. G. Craighead, P. Neuzil, C. Campagnolo, and C. Batt, J. Vac. Sci. Technol. B 19(6), 2825-2828 (2001).

${ }^{28}$ J. W. Yi, W. Y. Shih, R. Mutharasan, and W.-H. Shih, J. Appl. Phys. 93(1), 619-625 (2003)

${ }^{29}$ A. Mader, K. Gruber, R. Castelli, B. A. Hermann, P. H. Seeberger, J. O. Rädler, and M. Leisner, Nano Lett. 12(1), 420-423 (2012).

${ }^{30} \mathrm{~K}$. Y. Gfeller, N. Nugaeva, and M. Hegner, Appl. Environ. Microbiol. 71(5), 2626-2631 (2005)

${ }^{31}$ N. Nugaeva, K. Y. Gfeller, N. Backmann, M. Duggelin, H. P. Lang, H.-J. Guntherodt, and M. Hegner, Microsc. Microanal. 13(1), 13-17 (2007).

${ }^{32}$ N. Nugaeva, K. Y. Gfeller, N. Backmann, H. P. Lang, M. Düggelin, and M. Hegner, Biosens. Bioelectron. 21(6), 849-856 (2005).

${ }^{33}$ Y. Liu, L. M. Schweizer, W. Wang, R. L. Reuben, M. Schweizer, and W. Shu, Sens. Actuators B 178, 621-626 (2013).

${ }^{34}$ N. Maloney, G. Lukacs, N. Nugaeva, W. Grange, J. Ramseyer, J. Jensen, and M. Hegner, J. Sens. 2012, 405281 (2011)

${ }^{35}$ K. Y. Gfeller, N. Nugaeva, and M. Hegner, Biosens. Bioelectron. 21(3), 528-533 (2005)

${ }^{36}$ M. K. Ghatkesar, V. Barwich, T. Braun, J.-P. Ramseyer, C. Gerber, M. Hegner, H. P. Lang, U. Drechsler, and M. Despont, Nanotechnology 18(44), 445502 (2007).

${ }^{37}$ D. Ramos, J. Tamayo, J. Mertens, M. Calleja, L. Villanueva, and A. Zaballos, Nanotechnology 19(3), 035503 (2008).

${ }^{38}$ D. Ramos, J. Tamayo, J. Mertens, M. Calleja, and A. Zaballos, J. Appl. Phys. 100(10), 106105 (2006)

${ }^{39}$ J. Tamayo, D. Ramos, J. Mertens, and M. Calleja, Appl. Phys. Lett. 89(22), 224104 (2006). 